\title{
Accurate and scalable graph neural network force field and molecular dynamics with direct force architecture
}

\author{
Cheol Woo Park ${ }^{1,2}$, Mordechai Kornbluth (iD), Jonathan Vandermause $\mathbb{D}^{3}{ }^{3}$, Chris Wolverton (D) $^{2}$, Boris Kozinsky (iD ${ }^{1,3 凶}$ and \\ Jonathan P. Mailoa (iD) ${ }^{1 凶}$
}

Recently, machine learning $(\mathrm{ML})$ has been used to address the computational cost that has been limiting ab initio molecular dynamics (AIMD). Here, we present GNNFF, a graph neural network framework to directly predict atomic forces from automatically extracted features of the local atomic environment that are translationally-invariant, but rotationally-covariant to the coordinate of the atoms. We demonstrate that GNNFF not only achieves high performance in terms of force prediction accuracy and computational speed on various materials systems, but also accurately predicts the forces of a large MD system after being trained on forces obtained from a smaller system. Finally, we use our framework to perform an MD simulation of $\mathrm{Li}_{7} \mathrm{P}_{3} \mathrm{~S}_{11}$, a superionic conductor, and show that resulting Li diffusion coefficient is within 14\% of that obtained directly from AIMD. The high performance exhibited by GNNFF can be easily generalized to study atomistic level dynamics of other material systems.

npj Computational Materials (2021)7:73; https://doi.org/10.1038/s41524-021-00543-3

\section{INTRODUCTION}

In the past few decades, molecular dynamics (MD) has been extensively used to study and understand a wide range of chemical/physical phenomena at the atomistic level. The movement of the atoms in an MD simulation is dictated by the atomic forces obtained as gradients of the potential energy surface (PES) of the system. A common way of obtaining the PES is to explicitly calculate the electronic structure of the simulated system through ab initio approaches; density functional theory (DFT) is one of the methods in this category balancing speed and accuracy ${ }^{1-3}$. While ab initio MD (AIMD) provides highly accurate dynamics for many different atomic systems, the high computational cost of calculating the electronic structure derived forces places a hard limit on the system size (number of atoms) and duration (timesteps) of the simulation.

An alternative to AIMD is classical MD where the PES of an atomic system is constructed using interatomic potentials that define the interactions between atoms under specific bonding environments. Because it bypasses the need to calculate the electronic structure of the simulated system, classical MD has a significantly higher computational speed than AIMD and can be used to simulate larger systems for longer durations. Over the years, many different interatomic potentials have been developed to describe different bonding environments ${ }^{4-16}$ such as the Buckingham potential ${ }^{17}$ that describes ionic systems or the embedded-atom method ${ }^{4}$ that describes metallic systems. However, each potential is generally constrained to the one or several bond types that it was designed for, making these potentials unsuitable for simulating systems that have diverse and dynamic bonding environments often occurring in many important applications such as batteries and fuel cells ${ }^{18-20}$. While interatomic potentials like the reactive force field (ReaxFF) ${ }^{13}$ are actively being developed to simulate such complicated bonding environments, these methods, in their current states, are still limited by accuracy $^{21,22}$.
To address the limitations of both classical and ab initio MD, there has been an increasing interest in using machine learning $(\mathrm{ML})$ to generate $\mathrm{ab}$ initio quality $\mathrm{MD}$ with the computational efficiency comparable to that of classical $\mathrm{MD}^{23-31}$. The majority of the current state-of-the-art ML models use a set of features, often referred to as "atomic fingerprints," to represent the local environments of atoms that constitute the system of interest $^{23,32-38}$. These features are generally rotationally- and translationally-invariant, i.e., they remain constant under arbitrary rotational or translational transformations of the coordinate space. The atomic fingerprints are used to predict the PES of the system prior to taking its spatial derivative to obtain the atomic forces. The fingerprints are derived from manually designed functions that take as input the position of the center atom for which the fingerprint is being computed and the positions of neighboring atoms that are within a certain proximity of the center atom ${ }^{23,33}$.

Although the computational efficiencies of these ML models are better than ab initio methods, the cost of using these models in practice is still significantly high due to the computational bottlenecks that come from (1) deriving the atomic fingerprints needed for PES predictions and (2) calculating the derivatives of the PES to obtain forces ${ }^{33,39}$. More recently developed models address one or the other of these computational bottlenecks, but not both. In one approach, models bypass the need to design and compute the atomic fingerprints by utilizing deeper neural network architectures, similar to convolution or graph neural networks ${ }^{40,41}$, to automatically extract structural information of a material system ${ }^{28-31,38,42-45}$. For example, SchNet ${ }^{28,30}$ uses a series of continuous-filter convolutional layers to extract features from an arbitrary molecular geometry that can then be used to predict the PES of the molecule. While these models have shown excellent accuracies of atomic forces in single molecule systems, these models still rely on taking the derivatives of the PES to predict the atomic forces. In a different approach, the Direct Covariant Forces (DCF) model ${ }^{39}$ proposes an ML framework that predicts atomic forces directly using atomic fingerprints without having to take the derivatives of the PES. However, because the

\footnotetext{
${ }^{1}$ Robert Bosch Research and Technology Center, Cambridge, MA 02139, USA. ${ }^{2}$ Northwestern University, Evanston, IL 60208, USA. ${ }^{3}$ Harvard School of Engineering and Applied
}

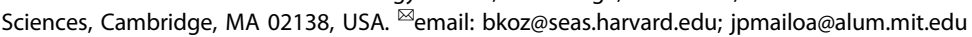




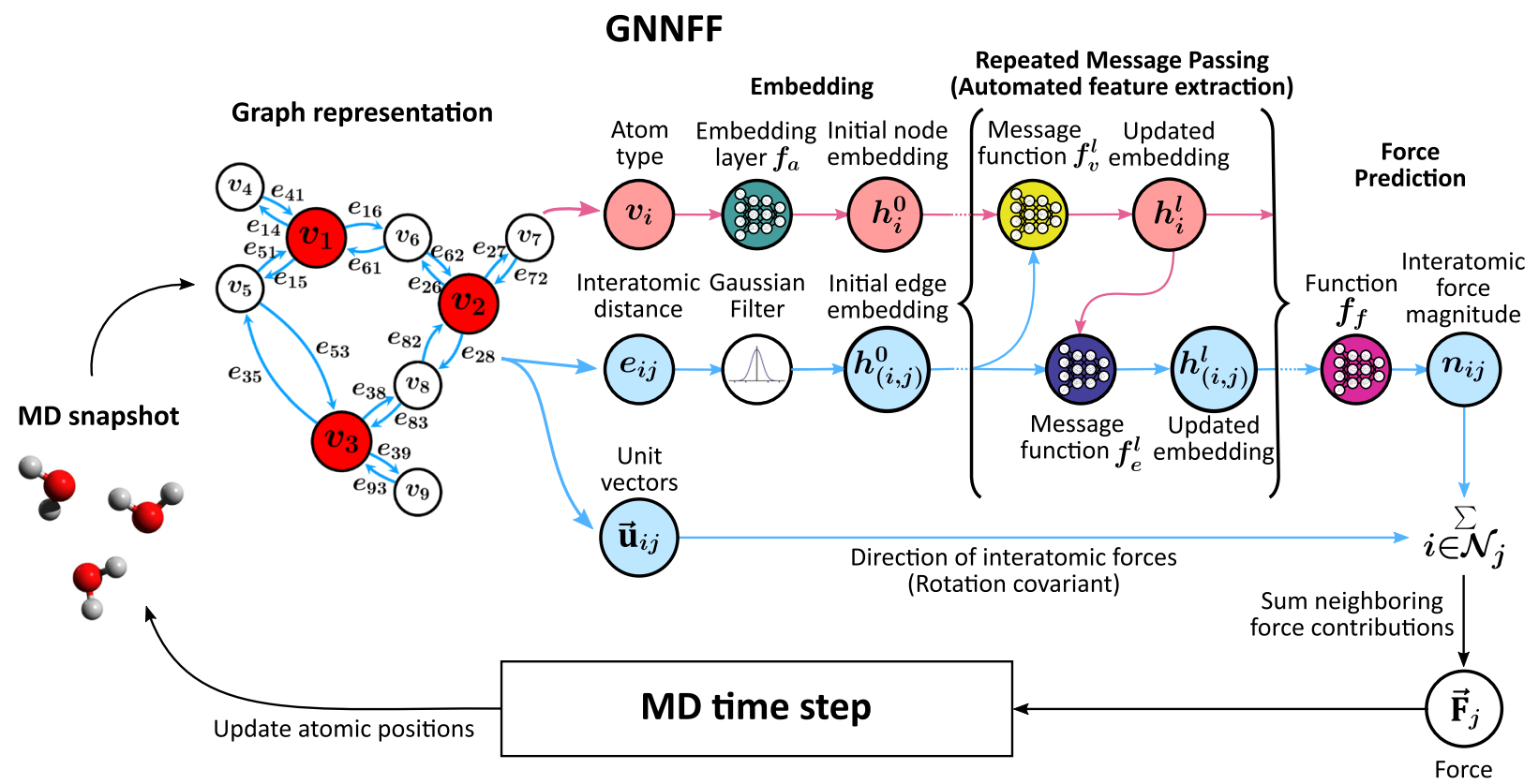

Fig. 1 Illustration of the GNNFF architecture. Each atomic configuration is represented as a directed graph that is then used as the input for GNNFF. Atom $i$ is represented as node $v_{i}$ and the influence atom $i$ has on atom $j$ is represented by a directed edge $e_{i j}$. The direction of this influence is represented by unit vector $\overrightarrow{\mathbf{u}}_{i j}$. Nodes and edges are embedded with latent vectors in the embedding stage. Initially, the node and edge embeddings respectively contain the atom type and interatomic distance information. The embeddings are then iteratively updated during the message passing stage. The final updated edge embeddings are used for predicting the interatomic force magnitudes. The force on the center atom $j$ is calculated by summing the force contributions of neighboring atoms $i \in \mathcal{N}_{j}$ that are calculated by multiplying the force magnitude and the respective unit vector. The predicted forces are finally used for updating the atomic positions in MD.

model relies on human-engineered atomic fingerprints, its prediction accuracy is limited.

In this study, we present a graph neural network force field (GNNFF) framework that bypasses both computational bottlenecks by predicting atomic forces directly using automatically extracted structural features that are translationally-invariant, but rotationally-covariant to the coordinate space of the atomic positions. We first demonstrate the accuracy and speed of GNNFF in calculating the atomic forces of various material systems against state-of-the-art models and benchmarks developed for each of those systems. For the first benchmark system of organic molecules found in the ISO17 database $28,29,46$, we compare GNNFF against the SchNet architecture. GNNFF outperformed SchNet by $16 \%$ in force prediction accuracy and by a factor of $1.6 \times$ in prediction speed. For the second benchmark system consisting of two multi-element solid-state systems (amorphous $\mathrm{Li}_{4} \mathrm{P}_{2} \mathrm{O}_{7}$ and $\mathrm{Al}_{2} \mathrm{O}_{3}$ in contact with high-concentration $\mathrm{HF}$ gas at the surface), a class of materials often neglected in ML tests, we benchmarked GNNFF against the DCF architecture ${ }^{39}$. Depending on the element-type and the system, GNNFF outperformed DCF by up to $30 \%$ on force prediction accuracy (up to $6.2 \times$ higher accuracy for rare chemical reaction events particularly difficult for DCF) and $4.5 \times$ on prediction speed.

We then further characterize the practical aspects of GNNFF's performance which are based on (1) scalability and (2) the physical/chemical accuracy of the GNNFF generated trajectories. Scalability measures how accurately the ML model can predict the forces of a system after being trained on forces obtained from a smaller system. For this assessment, we generated two separate AIMD trajectories of $\mathrm{Li}_{7-x} \mathrm{P}_{3} \mathrm{~S}_{11}$, a superionic conducting material. One uses a $1 \times 2 \times 1$ supercell (Small) and the other a $1 \times 2 \times$ 2 supercell (Large), each with a single Li-ion vacancy compensated by a background charge. When trained solely on the forces taken from the "Small" supercell, we show that GNNFF is able to predict the forces of the "Large" system as accurately as it can predict the forces of the "Small" system where the differences in accuracies in predicting the forces were within 3\%. To illustrate the physical/ chemical accuracy of the GNNFF generated trajectories, we measured the Li-ion diffusion coefficient from an ML-driven simulation of $\mathrm{Li}_{7-x} \mathrm{P}_{3} \mathrm{~S}_{11}$ performed under the same conditions as the AIMD "Small" simulation using the previously trained GNNFF. The diffusivity measured from GNNFF-generated trajectory was within $14 \%$ relative to that measured directly from the AIMD 'Small' trajectory. The versatility and excellent performance exhibited by GNNFF strongly suggest that it can be used to effectively accelerate many studies of atomistic level dynamics through MD.

\section{RESULTS}

\section{The Graph Neural Network Force Field (GNNFF) model}

The general architecture of GNNFF is illustrated in Fig. 1, and is inspired by previous work on crystal graph convolutional neural networks ${ }^{31,45}$. First, the atomic structure of each MD snapshot is represented as a directed graph $\mathcal{G}=(\mathcal{V}, \mathcal{E}, \mathcal{U})$, with node $v_{i} \in \mathcal{V}$ representing the constituent atom $i$, directed edge $e_{i j} \in \mathcal{E}$ representing the influence atom $i$ has on neighboring atom $j$, and $\overrightarrow{\mathbf{u}}_{i j} \in \mathcal{U}$ representing the unit vector pointing from atom $i$ to atom $j$. Here, we emphasize that the edges are directed where $e_{i j} \neq e_{j i}$ implying that the influence that atom $i$ has on atom $j$ is not the same as the influence that atom $j$ has on atom $i$. Each node is connected to its $N$ closest neighbors where $N$ is a predefined number. The graph representation is used as input for GNNFF which is composed of the embedding, the message-passing, and the force vector calculation stages.

In the embedding stage, node $v_{i}$ and edge $e_{i j}$ are embedded with latent vectors to represent the hidden physical/chemical states of the atom and bond respectively, where we define embedding to be the mapping of a discrete object to a vector of real numbers. Initial node embedding $h_{i}^{0}$ is the output of a neural network where the input is a one-hot (categorical) vector 
representing the element-type of atom $i$. The purpose of using one-hot vector representations is purely to differentiate one element-type from another. For example, in a system that consists only of water, hydrogen and oxygen can be respectively represented as one-hot vectors [1, 0] and [0, 1]. The initial edge embedding $h_{(i, j)}^{0}$ is given by expanding the distance between atoms $i$ and $j$ in a Gaussian basis, a process we refer to as Gaussian filtering. Mathematically this can be expressed as:

$f_{a}:$ One $-\operatorname{Hot}($ Type $($ atom $i)) \rightarrow h_{i}^{0}$

$G: d(i, j) \rightarrow h_{(i, j)}^{0}$

where $f_{a}$ denotes the neural network that maps the one-hot encoding of the element-type of atom $i$ to $h_{i}^{0}, G$ denotes the Gaussian filtering operation, and $d(i, j)$ denotes the distance between atoms $i$ and $j$.

The message-passing stage of GNNFF consists of multiple layers where in each layer, "local messages" are passed between neighboring nodes and edges. We define the local message passing that occurs between the nodes and edges as follows:

Edge to node $: f_{v}^{\prime}:\left\{h_{i}^{\prime}, h_{(i, j)}^{\prime} \mid j \in \mathcal{N}_{i}\right\} \rightarrow h_{i}^{l+1}$

Node to edge $: f_{e}^{l}:\left\{h_{i}^{l+1}, h_{j}^{l+1}, h_{(i, j)}^{\prime}\right\} \rightarrow h_{(i, j)}^{l+1}$

$h_{i}^{\prime}$ and $h_{(i, j)}^{\prime}$ are the embeddings of node $v_{i}$ and edge $e_{i j}$ in the message-passing layer $l$, respectively. Initial conditions for $h_{i}^{l}$ and $h_{(i, j)}^{\prime}$ are given by $h_{i}^{0}$ and $h_{(i, j)}^{0}$ which were determined in the embedding stage. $\mathcal{N}_{i}$ denotes the set of indices of neighboring nodes connected to node $v_{i}$. Message functions $f_{v}^{l}$ and $f_{e}^{\prime}$ are nodeand edge- specific neural networks that update $h_{i}^{\prime}$ and $h_{(i, j)}^{\prime}$ to $h_{i}^{l+1}$ and $h_{(i, j)}^{1+1}$ respectively. These functions are defined such that after each update (message-passing), embedding $h_{i}^{l}$ better represents the local atomic environment of atom $i$ and embedding $h_{(i, j)}^{\prime}$ better represents the interatomic interactions between atoms $i$ and $j$. The designs for these functions are flexible and can be altered to emphasize the different interatomic interactions that occur within MD simulations. In the current implementation of GNNFF, we defined the functions to be the following:

$$
\begin{aligned}
& f_{v}^{\prime}: h_{i}^{\prime+1}=\tanh \\
& {\left[h_{i}^{\prime}+\sum_{j \in \mathcal{N}_{i}} \sigma\left(\mathbf{c} 1_{i j}^{\prime} \boldsymbol{W}_{1}^{\prime}+\boldsymbol{b}_{1}^{\prime}\right) \odot \tanh \left(\mathbf{c} 1_{i j}^{\prime} \boldsymbol{W}_{2}^{\prime}+\boldsymbol{b}_{2}^{\prime}\right)\right]} \\
& f_{e}^{\prime}: h_{(i, j)}^{l+1}=\tanh \\
& {\left[\begin{array}{c}
h_{(i, j)}^{\prime}+\sigma\left(\mathbf{c 2} 2_{i j}^{\prime} \boldsymbol{W}_{3}^{\prime}+\boldsymbol{b}_{3}^{\prime}\right) \odot \tanh \left(\mathbf{c} 2_{i j}^{\prime} \boldsymbol{W}_{4}^{\prime}+\boldsymbol{b}_{4}^{\prime}\right) \\
+\sum_{k \in \mathcal{N}_{j}} \sigma\left(\mathbf{c} 3_{i j k}^{\prime} \boldsymbol{W}_{5}^{\prime}+\boldsymbol{b}_{5}^{\prime}\right) \odot \tanh \left(\mathbf{c} 3_{i j k}^{\prime} \boldsymbol{W}_{6}^{\prime}+\boldsymbol{b}_{6}^{\prime}\right)
\end{array}\right]}
\end{aligned}
$$

where $\sigma$ denotes a sigmoid function that is applied element-wise to each entry of the vector and $\odot$ denotes an element-wise multiplication operator. $\boldsymbol{W}^{\prime}$ and $\boldsymbol{b}^{\prime}$ represent the weights and biases of the neural network hidden layers that compose the message-passing layer '. $c 1_{i j}^{1}$ represents how the interatomic dynamic between atoms $i$ and $j$ impacts the local environment of atom $i$ and is defined as $\mathbf{c} 1_{i j}^{\prime}=\boldsymbol{h}_{i}^{\prime} \oplus \boldsymbol{h}_{(i, j)}^{\prime}$, where $\oplus$ denotes a concatenation operator. $\mathbf{c} 2_{i j}^{l}$ and $\mathbf{c} 3_{i j k}^{l}$ respectively represent the 2 body correlation between atoms $i$ and $j$ and 3-body correlation between atoms $i, j$, and $k$, where $\quad \mathbf{c 2}_{i j}^{l}=\boldsymbol{h}_{i}^{\prime} \odot \boldsymbol{h}_{j}^{\prime}$ and c3 $_{i j k}^{\prime}=\boldsymbol{h}_{i}^{\prime} \oplus \boldsymbol{h}_{j}^{\prime} \oplus \boldsymbol{h}_{k}^{\prime} \oplus \boldsymbol{h}_{(i, j)}^{\prime} \oplus \boldsymbol{h}_{(k, j)}^{\prime}$. We note that the 2-body and 3-body correlation terms use different operators. These operators were chosen based on trial-and-error where we found that using the $\odot$ operator for the 2-body correlation term and $\oplus$ operator for the 3-body correlation term yielded the best ML performance on the validation set. The many-body correlations beyond the 2-body and 3-body contributions are implicitly captured by repeatedly passing messages between the nodes and edges. However, this can also result in the loss of information as the message travels further away from its point of origin. To minimize this loss, Eq. (5) and (6). use an activation function $g$ to normalize information after each message-passing iteration and an activation function $\sigma$ to act as a gate that allows only the most relevant information to pass through ${ }^{47}$.

The use of latent vectors that are iteratively updated instead of human-engineered atomic fingerprints to represent the chemical/ physical state of the local atomic environment in itself is not a new practice $28,30,31,45$. However, GNNFF is different from existing models in how it uses the updated edge embeddings to calculate force vectors that are rotationally-covariant to the coordinate space in the final stage. For a GNNFF with $L$ message-passing layers, the final updated state of edge $e_{i j}$, represented by embedding $h_{(i, j)}^{L}$, is used to calculate $n_{i j}$, a scalar quantity that denotes the magnitude of the force contribution that atom $i$ is exerting on atom $j$. The force contribution of atom $i$ onto $j$ is given by $n_{i j} \overrightarrow{\mathbf{u}}_{i j}$ and the total force prediction on atom $j$ is simply the vector sum of the force contributions of all neighboring atoms. Mathematically the individual force contribution and the total force prediction can respectively be written as:

$f_{f}: h_{(i, j)}^{L} \rightarrow n_{i j}$

$\overrightarrow{\mathbf{F}}_{j}=\sum_{i \in \mathcal{N}_{j}} n_{i j} \overrightarrow{\mathbf{u}}_{i j}$

where the function $f_{f}$ is a neural network in the final stage that maps $h_{(i, j)}^{L}$ to $n_{i j}$ and $\overrightarrow{\mathbf{F}}_{j}$ is the force prediction on atom $j$. Because $\overrightarrow{\mathbf{F}}_{j}$ is a linear function of $\overrightarrow{\mathbf{u}}_{i j} \in \mathcal{U}$, which is inherently translationally-invariant and rotationally-covariant to the input coordinates of the atoms, the GNNFF-predicted forces are translationally-invariant and rotationally-covariant to the input atomic coordinates environment $\left\{\overrightarrow{\mathbf{R}}_{t}\right\}$ of the MD snapshots as well. Also, since we only consider the force contributions of a fixed number of neighboring atoms, the computational cost of using GNNFF to predict new forces scales linearly with the size of the system. The weights and biases in $f_{a}, f_{v}^{\prime}, f_{e^{\prime}}^{\prime}$ and $f_{f}$ are shared across all the atoms.

\section{Comparing GNNFF performance to other models}

In this section, we characterize the performance of GNNFF in predicting atomic forces through two different illustrations. First, we evaluate GNNFF performance in predicting the forces of simple organic molecules in reference to SchNet which provides one of the best published benchmarks available for predicting forces of single-molecule $M D^{28,30}$. Subsequently, GNNFF is evaluated on complex solid-state systems in reference to DCF, which has been trained and tested for predicting forces of complicated multielement solid-state systems ${ }^{39}$.

To compare the GNNFF performance in predicting the forces of organic molecules to that of SchNet, GNNFF was trained and tested in the same way SchNet was evaluated in ref. ${ }^{28}$. The organic molecule force data used for evaluation were taken from the ISO17 database $28,29,46$, a collection of MD trajectories simulated for 129 organic isomers, all with the composition of $\mathrm{C}_{7} \mathrm{O}_{2} \mathrm{H}_{10}$, but with distinct structures. The trajectory for each isomer consists of 5000 snapshots, resulting in a total of 645,000 unique snapshots. The snapshots are divided into three nonoverlapping sets: one training set and two test sets. The training set consists of $80 \%$ of the snapshots randomly selected from the MD trajectories of $80 \%$ of the 129 isomers. The first test set, referred to as the 'test within' set, consists of the remaining $20 \%$ of the snapshots obtained from the same molecules used in the training set. The second and more challenging test set consists of 
Table 1. Comparison of GNNFF and SchNet Cartesian force components predictive accuracy of the ISO17 database.

\begin{tabular}{llll}
\hline Test set & $\left\langle\left|\overrightarrow{\mathbf{F}}_{\mathrm{DFT}}\right|\right\rangle\left(\mathrm{eV} \AA^{-1}\right)$ & $\mathrm{MAE}\left(\mathrm{eV} \AA^{-1}\right)$ & \\
\cline { 3 - 4 } & & $\mathrm{SchNe{ } ^ { 2 8 }}$ & $\mathrm{GNNFF}$ \\
\hline Test within & 1.698 & 0.043 & 0.036 \\
Test other & 1.664 & 0.095 & 0.088 \\
\hline
\end{tabular}

all the snapshots taken from the other $20 \%$ of the 129 molecules that are not included in the training set and is referred to as the "test other" set. The purpose of the first test set is to evaluate an ML model's ability to interpolate the forces of unknown geometries for known molecules, i.e., molecules that are present in the training data, while the second test set evaluates the ability of GNNFF to generalize to unknown molecules, i.e., molecules that were never encountered in training. Predictive accuracies are evaluated based on the mean absolute error (MAE) of the Cartesian force components. The test results are summarized in Table 1. For "test within", MAE of GNNFF was $0.036 \mathrm{eV} \AA^{-1}$ which is $16 \%$ lower than that of SchNet $\left(0.043 \mathrm{eV}^{-1}\right)$ indicating that GNNFF is better at capturing chemical/structural features of a molecular conformation necessary for interpolating the forces of unknown conformations for known molecules, i.e., molecules that are included in the training set. For "test other", GNNFF MAE of $0.088 \mathrm{eV} \AA^{-1}$ was $7 \%$ lower compared to that of SchNet $(0.095 \mathrm{eV}$ $\AA^{-1}$ ) implying that GNNFF extracted chemical/structural features are more generalized and therefore, better suited for inferring the forces of new unknown molecules that the ML model never encountered in training.

We also evaluated the computational efficiency of SchNet and GNNFF in predicting atomic forces of the "test within" and "test other" data sets. Evaluations were performed on a workstation equipped with an Nvidia GTX 1080 GPU and an Intel i7-8700K 6core $3.70 \mathrm{GHz}$ CPU processor. The efficiency of SchNet was evaluated using the SchNetPack code that is available through github.com/atomistic-machine-learning/schnetpack. The average time for SchNet to evaluate the forces of the MD snapshots in the "test within" and 'test other' sets was $10.5 \times 10^{-4} \mathrm{~s}$ per atom per snapshot. For GNNFF, the evaluation time was $6.1 \times 10^{-4} \mathrm{~s}$ per atom per snapshot, indicating that GNNFF is $\sim 1.6 \times$ faster. While various factors can be attributed to this speed up such as code optimization or the size of the neural networks, these results suggest that GNNFF is faster than SchNet in its out-of-the-box state and demonstrate that GNNFF can be effectively used for accelerating the chemical exploration of organic molecules with performance on par with current state-of-the-art models.

Here, we benchmark the GNNFF performance to that of DCF in predicting the atomic forces of the AIMD trajectories of two different solid-state systems. The first system is $\mathrm{Li}_{4} \mathrm{P}_{2} \mathrm{O}_{7}$ at a temperature $T=3000 \mathrm{~K}$, well beyond its melting temperature. Temperature was regulated with a Nosé-Hoover thermostat and MD was performed with a timestep of $2 \mathrm{fs}$ for 50 ps yielding a total of $\sim 25,000$ snapshots. This simulation represents part of the rapid annealing process that turns crystalline $\mathrm{Li}_{4} \mathrm{P}_{2} \mathrm{O}_{7}$ into an amorphous state. Using this system, we evaluate GNNFF's ability to learn and recognize the subtle differences between the different phases of the oxide that are present in the simulation. The second system involves $\mathrm{Al}_{2} \mathrm{O}_{3}$ in contact with $\mathrm{HF}$ molecules at $T=1000 \mathrm{~K}$ where chemical reactions occur between the HF acids and the $\mathrm{Al}$ atoms. Temperature was regulated with a Nosé thermostat and a timestep of $0.5 \mathrm{fs}$ was used with a total duration of $\sim 7 \mathrm{ps}$ yielding $\sim 13,000$ snapshots. This system is used for evaluating GNNFF's performance in capturing dynamics that drive chemical reactions.

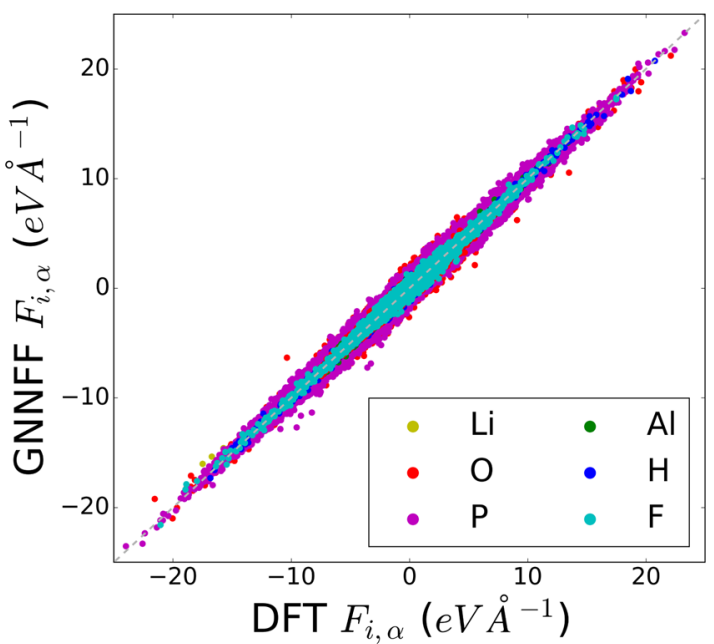

Fig. 2 Performance of GNNFF in predicting forces of solid-state systems. GNNFF-predicted vs DFT-calculated force correlation plots of elements from the $\mathrm{Li}_{4} \mathrm{P}_{2} \mathrm{O}_{7}$ and $\mathrm{Al}_{2} \mathrm{O}_{3}-\mathrm{HF}$ MD systems. $F_{i, a}(a \in\{x, y$, $z\})$ represents the Cartesian force component of atom $i$.

The $\mathrm{Li}_{4} \mathrm{P}_{2} \mathrm{O}_{7}$ and $\mathrm{Al}_{2} \mathrm{O}_{3}-\mathrm{HF}$ systems consist of 208 and 228 atoms respectively, both significantly larger than the isomers of ISO17. Detailed explanations on the generation of these simulations can be found in ref. ${ }^{39}$. GNNFF was trained and tested separately for each MD trajectory where the snapshots were shuffled and divided into 80 and $20 \%$ of the total data to form the training set and test set. For the $\mathrm{Li}_{4} \mathrm{P}_{2} \mathrm{O}_{7}$ system, the training data consisted of $\sim 20,000$ snapshots and the testing data consisted of $\sim 5000$ snapshots. For the $\mathrm{Al}_{2} \mathrm{O}_{3}$-HF system, there were $\sim 11,000$ training and $\sim 3000$ testing snapshots. Similar to the DCF evaluation criteria ${ }^{39}$, the GNNFF performance was quantified separately for each element-type $e$ by the ratio of the vector mean absolute error (vMAE) between the GNNFF-predicted forces ( $\overrightarrow{\mathbf{F}}_{\text {GNNF.e }}$ ) and DFTcalculated forces $\left(\overrightarrow{\mathbf{F}}_{\mathrm{DFT}, e}\right)$, to the mean absolute value (MAV) of the DFT-calculated forces. Specifically, prediction accuracy is given by

$\frac{\mathrm{VMAE}_{e}}{\mathrm{MAV}_{e}}=\frac{\left\langle\left|\overrightarrow{\mathbf{F}}_{\mathrm{DFT}, e}-\overrightarrow{\mathbf{F}}_{\mathrm{GNNFF}_{, e}}\right|\right\rangle}{\left\langle\left|\overrightarrow{\mathbf{F}}_{\mathrm{DFT}, e}\right|\right\rangle}$

a metric that is comparable across temperatures and elements where the brackets indicate an averaging operation. The numerator averages the absolute differences between the DFTcalculated and GNNFF-predicted forces for element-type $e$ while the denominator averages the magnitude of the DFT-calculated forces of $e$. Lower $\frac{\mathrm{VMAE}_{e}}{\mathrm{MAV}_{e}}$ indicates higher predictive accuracy. We note that this is different from the MAE of the Cartesian force components used previously to compare GNNFF and SchNet.

As shown in Fig. 2, the correlations between the ML-predicted and DFT-calculated forces are clearly evident for all elements in both systems, implying the high general performance of GNNFF in predicting forces. The predictive accuracy of GNNFF for each element is summarized in Table 2 and compared to that of DCF. The improvement of GNNFF over DCF measured in vMAE/MAV \% values ranges from $8 \%$ for the lighter elements such as $\mathrm{H}, \mathrm{O}$, and $\mathrm{F}$ to $30 \%$ for the heavier elements such as $\mathrm{P}$ and Al.

The time for GNNFF to evaluate the test sets for the $\mathrm{Li}_{4} \mathrm{P}_{2} \mathrm{O}_{7}$ and $\mathrm{Al}_{2} \mathrm{O}_{3}$ - $\mathrm{HF}$ systems were both $1.8 \times 10^{-3} \mathrm{~s}$ per atom per snapshot on a single core of a high-performance cluster mounted with $2.10 \mathrm{GHz}$ Intel Xeon Gold 6230 processor. Using the same hardware setup, DCF took $7.8 \times 10^{-3}$ and $8.8 \times 10^{-3} \mathrm{~s}$ per atom per snapshot to evaluate the $\mathrm{Li}_{4} \mathrm{P}_{2} \mathrm{O}_{7}$ and $\mathrm{Al}_{2} \mathrm{O}_{3}-\mathrm{HF}$ test sets respectively, showing that GNNFF is on average $4.5 \times$ faster than 
DCF in predicting forces. These results again demonstrate GNNFF's competitive edge against existing state-of-the-art models.

Using the $\mathrm{Al}_{2} \mathrm{O}_{3}-\mathrm{HF}$ system, we further investigated the GNNFF's performance in predicting the atomic forces involved in rare events as was done in ref. ${ }^{39}$. The trajectory contains chemical reactions where a $\mathrm{HF}$ molecule associates and dissociates from an $\mathrm{Al}$ atom at the surface of $\mathrm{Al}_{2} \mathrm{O}_{3}$ as shown in Fig. 3(a). The snapshots that correspond to the events in which the HF molecules react with the $\mathrm{Al}$ atoms, or simply the "reaction snapshots", occur on average once every 8 time steps and are extracted from the trajectory according to a Hidden Markov Model (HMM) as explained in the Methods section.

Table 2. Comparing the accuracies of GNNFF and DCF in predicting the atomic forces of solid-state systems, $\mathrm{Li}_{4} \mathrm{P}_{2} \mathrm{O}_{7}$ and $\mathrm{Al}_{2} \mathrm{O}_{3}-\mathrm{HF}$.

\begin{tabular}{llll}
\hline System & Atom & \multicolumn{2}{l}{ vMAE/MAV (\%) } \\
\cline { 3 - 4 } & & DCF & GNNFF \\
\hline $\mathrm{Li}_{4} \mathrm{P}_{2} \mathrm{O}_{7}$ & $\mathrm{Li}$ & 37 & 13 \\
& $\mathrm{O}$ & 25 & 10 \\
& $\mathrm{P}$ & 40 & 10 \\
$\mathrm{Al}_{2} \mathrm{O}_{3}-\mathrm{HF}$ & $\mathrm{Al}$ & 33 & 14 \\
& $\mathrm{~F}$ & 22 & 14 \\
& $\mathrm{H}$ & 22 & 14 \\
& $\mathrm{O}$ & 35 & 15 \\
\hline
\end{tabular}

Accuracy is measured separately for each element in terms of vMAE/MAV, where lower values indicate higher accuracies. For both systems, GNNFF accuracy for each element is higher than that of DCF.
While the sparsity of data makes it especially difficult to train an $\mathrm{ML}$ model to correctly predict the atomic forces of reacting snapshots, it is nonetheless important to quantify an ML model's performance in this regard as these rare occurrences are generally the phenomena we would be interested in studying with MLdriven MD. For our assessment, we trained another GNNFF model from scratch using only the nonreacting snapshots and tested it using the reacting snapshots (reaction test set). There were $\sim 1600$ reacting snapshots leaving $\sim 12,400$ snapshots for training.

We compared the force prediction accuracy of GNNFF on the reaction test set to our previous evaluation where snapshots of the $\mathrm{Al}_{2} \mathrm{O}_{3}-\mathrm{HF}$ were randomly shuffled prior to being divided into training and testing data (standard testing set) as reported in Table 2 . Figure $3 b$ shows that the vMAE/MAV \% values for each element-type measured on the reaction and standard test set differ by $5 \%$ or less. According to the HMM that is used for extracting the reaction snapshots, oxygen is occasionally recognized as part of the alumina surface and HF reaction when the HF acid interacts with a unit of $\mathrm{Al}-\mathrm{O}$ rather than the individual $\mathrm{Al}$ atom. However, the reaction snapshots corresponding to these reaction events involving oxygen comprise less than $\sim 0.1 \%$ of the total trajectory, making force predictions of oxygen in the reaction test set a challenge ${ }^{39}$. In Fig. $3 c$, the correlation between the GNNFF-predicted and DFT-calculated forces of the reaction test set is as clear as that of the standard test set. The GNNFF vMAE/ MAV \% value for oxygen in the reaction test set was $17 \%$. The corresponding value reported for DCF is $6.2 \times$ higher at $105 \%$. We hypothesize that this significant improvement happens because GNNFF is a fully rotationally-covariant force prediction algorithm that does not require hand-crafted descriptors, while DCF is only trained to be rotationally-covariant using data augmentation and relies on manually designed descriptors.

(a)
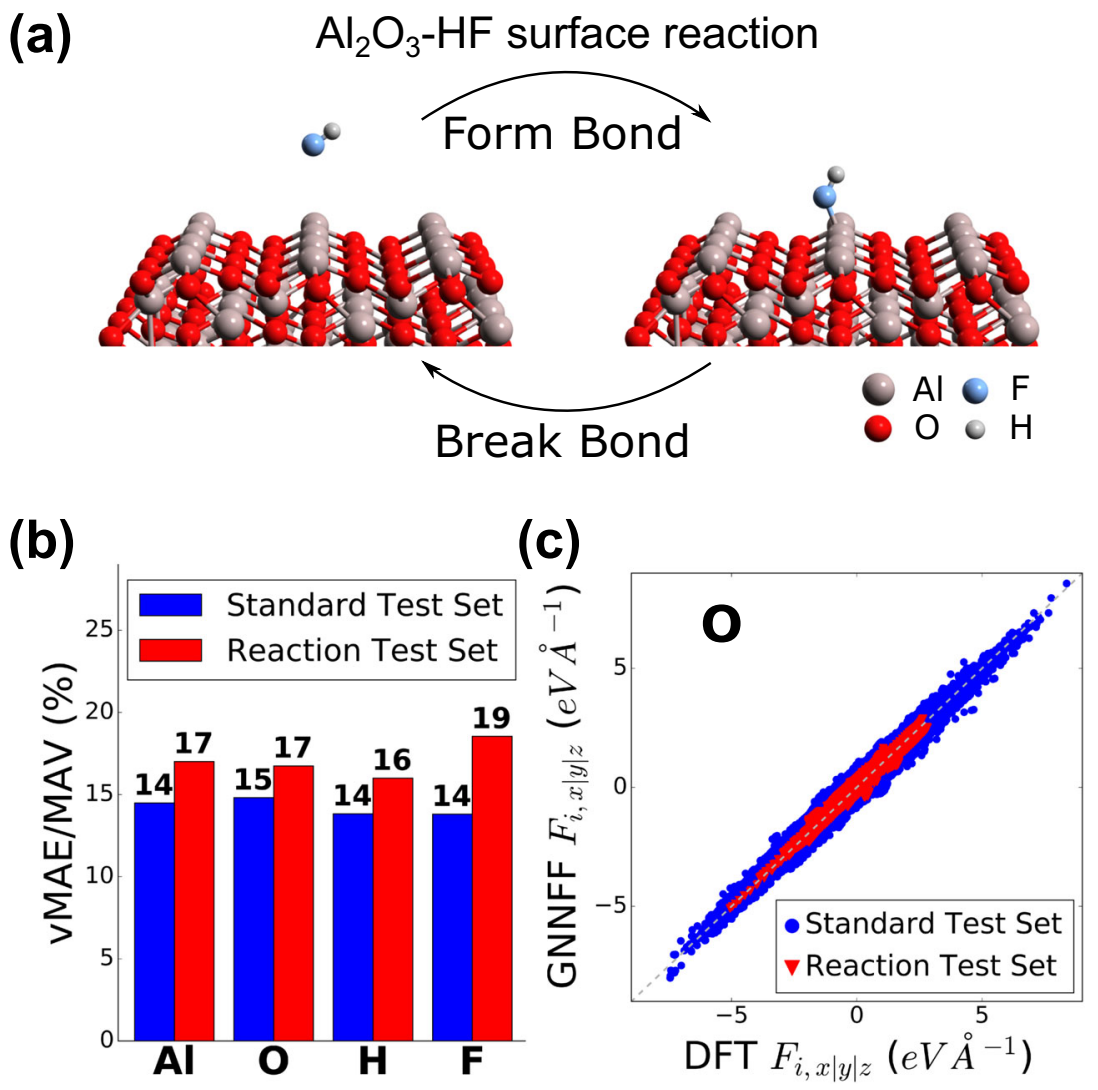

(c)

Fig. 3 Predicting the forces on atoms involved in surface reaction between $\mathbf{A l}_{2} \mathbf{O}_{3}$ and $\mathbf{H F}$. a Illustration of the $\mathrm{Al}_{2} \mathrm{O}_{3}-\mathrm{HF}$ surface reaction $\mathbf{b}$ Comparison of force predictive accuracies between standard and reaction test sets for all elements present in system. c GNNFF-predicted vs DFT-calculated force correlation plots of oxygen for both standard and reaction test sets. 


\section{Assessing the practical effectiveness of GNNFF}

Here we assess the practical effectiveness of GNNFF and begin by stating what the GNNFF cannot be used for. Because the forces of GNNFF are not derived from the PES of the system, they are not energy conserving. This is easily checked by performing an NVE simulation using GNNFF predicted forces, for which more details can be found in Supplementary Note 2. This implies that GNNFF cannot be used to run micro-canonical simulations or to measure properties that are related to energy of the system such as formation energy or transition barriers.

GNNFF, however, can still be used to run MD simulations in an NVT setting where a thermostat is used to regulate the temperature. Within an NVT setting, the expected effectiveness of GNNFF in practice can be further tested based on two additional criteria. The first criterion is scalability: how does the GNNFF perform when we use it to predict the forces of an MD system that is larger than the system used for generating the training data? Ab initio methods are limited by computational cost in simulating large materials systems with a lot of atoms. This can be problematic when studying physical phenomena such as nucleation, dislocation, and grain boundary formations which require sufficiently large $\mathrm{MD}$ systems in order to be observed. Having an ML model that can consistently provide accurate force predictions regardless of the MD system size would greatly expand our capability to explore diverse physical phenomena at the atomic level. The second criterion is how physically/chemically accurate the GNNFF generated simulations are in reference to AIMD $^{48,49}$. For example, if we are interested in studying the ionic conducting properties of a material using $\mathrm{ML}$, it is important that the ionic conductivity measured from the ML simulated trajectory to be close to that measured from AIMD.

Our system of choice to test GNNFF for these criteria is $\mathrm{Li}_{7} \mathrm{P}_{3} \mathrm{~S}_{11}$, a superionic conducting material. Recent study has shown that the conductivity of $\mathrm{Li}_{7} \mathrm{P}_{3} \mathrm{~S}_{11}$ at $300 \mathrm{~K}$ predicted by AIMD is $57 \mathrm{mS} \mathrm{cm}^{-1}$ which is about $5 \times$ greater than the experimentally measured 11.6 $\mathrm{mS} \mathrm{cm}^{-119}$. The lower experimentally measured conductivity is speculated to be due to grain boundaries which deter ion transport. Using AIMD to accurately determine the effect that grain boundaries (GB) have on ion transport is problematic, as it would require significantly larger systems than bulk (GB free) simulations. While a detailed investigation of ion dynamics in grain boundaries using $M L$ is beyond the scope of this study, $\mathrm{Li}_{7} \mathrm{P}_{3} \mathrm{~S}_{11}$ is a good example where having an ML model that satisfies the aforementioned scaling and accuracy criteria could greatly help in studying its physical/chemical properties.

Data used for this assessment was obtained from two separate AIMD trajectories of $\mathrm{Li}_{7-\mathrm{x}} \mathrm{P}_{3} \mathrm{~S}_{11}$. These trajectories differ in system size, where one uses a $1 \times 2 \times 1$ supercell ("Small") and the other uses a $1 \times 2 \times 2$ supercell ("Large"). Both systems contain a single $\mathrm{Li}^{+}$vacancy to accelerate ion diffusion resulting in 83 atoms $(x=$ $0.25)$ in the "Small" system and 167 atoms $(x=0.125)$ in the "Large" system. Simulations were performed at $520 \mathrm{~K}$ using a Nosé-Hoover thermostat. The "Small" and "Large" trajectories respectively consist of $\sim 25,000$ and $\sim 7500$ snapshots. More details of how these simulations were generated can be found in the "Methods" section.

To illustrate the scalability of GNNFF, we first trained it on 20,000 snapshots randomly selected from the "Small" trajectory. We then compared GNNFF accuracy in predicting the forces of all $\sim 7500$ snapshots provided by the 'Large' trajectory and the 5000 snapshots in the "Small" trajectory that were not included in the training data. Accuracy was again measured separately for each element-type in vMAE/MAV.

As shown in Fig. 4b, the differences in vMAE/MAV \% values for the "Small" and "Large" systems were within 3\% for all elements, demonstrating the consistency of the GNNFF force prediction accuracy on the "Large" trajectory even after being trained on the
"Small" trajectory forces. In Fig. 4c, the GNNFF-predicted vs DFTcalculated force correlation plots of the element $S$ for the "Large" and "Small" test sets are overlaid together. While the force correlation of the "Large" test set is as good as that of the "Small" test set, some of the ML-predicted values significantly deviate from the DFT-calculated values as indicated by the blue arrows. Although these deviations were only observed for the force predictions of $\mathrm{S}$, they could cause concern as they might lead to unphysical atomic configurations when using GNNFF to perform MD.

To address this concern, we performed an NVT MD simulation for the "Large" system size, but using atomic forces (and hence, evolution of the trajectory) calculated by the GNNFF trained on the 'Small' trajectory forces. We then compared atomic configurations of the GNNFF-simulated trajectory to the ab initio generated "Large" trajectory. For a fair comparison, GNNFFgenerated simulation was performed under the same conditions as its ab initio counterpart in terms of temperature, thermostat, timestep, simulation duration, and initial structure. Throughout the ML-driven simulation, no breaking of the P-S bonds was observed. The radial distribution functions (RDFs) and angular distribution functions (ADFs) of the GNNFF and AIMD trajectories are highly consistent with one another as shown respectively in Fig. 4d, e. This demonstrates that the average atomic configurations generated by GNNFF MD are physically reasonable despite the deviations in the force predictions observed in Fig. 4c.

To evaluate the physical/chemical accuracy of GNNFF generated trajectories, we calculate the diffusivity of Li-ions in $\mathrm{Li}_{7-x} \mathrm{P}_{3} \mathrm{~S}_{11}(x=$ 0.25 ) with an MD simulation using GNNFF-predicted forces and compare it with that obtained directly from AIMD. The ab initio value for the Li diffusivity was obtained from the AIMD "Small" trajectory that was simulated for $50 \mathrm{ps}$. The temperature of the MD simulation was regulated using a Nosé-Hoover thermostat with a time constant $t_{T}=27 \mathrm{fs}$. The effects of thermostat strength on $\mathrm{Li}$ diffusivity are discussed in detail in Supplementary Note 3. For an apples-to-apples comparison, we performed an MD simulation equivalent of the "Small" trajectory using the previously trained GNNFF to determine the ML predicted Li diffusivity. Again, no breaking of the P-S bond was observed throughout the GNNFFdriven simulation. In Fig. 5a, we compare the element-specific probability distribution function (PDF) of the force magnitudes in the GNNFF MD run and AIMD run. While there is an overall tendency of GNNFF to underestimate the force magnitudes compared to DFT, we generally see good agreement between the GNNFF and AIMD PDFs for all element-types. In Fig. 5(b), we compute and plot the mean square displacement (MSD) of Li-ions as a function of time lag $(\Delta r)$. The diffusivity of $\mathrm{Li}_{7-x} \mathrm{P}_{3} \mathrm{~S}_{11}(x=0.25)$ at $520 \mathrm{~K}$ determined from the MSD slope was $1.7 \times 10^{-5} \mathrm{~cm}^{2} \mathrm{~s}^{-1}$ for GNNFF which is in good agreement with the AIMD value of $1.5 \times 10^{-5} \mathrm{~cm}^{2} \mathrm{~s}^{-1}$. This demonstrates that the accuracy of the GNNFF-driven MD simulations is on par with that of the simulations generated through ab initio methods. With methods like GNNFF that are more scalable than AIMD, direct simulations of ion transport across defects and grain boundaries are more feasible and will be the subject of future work.

\section{DISCUSSION}

GNNFF provides a versatile framework to directly predict atomic forces from automatically extracted features of the local atomic environment that are rotationally-covariant to the coordinate space. This enables GNNFF to bypass both computing atomic fingerprints and calculating the derivatives of the PES which are often the computational bottlenecks of existing $\mathrm{ML}$ force field models.

In this work, we demonstrated the competitive edge of GNNFF in terms of accuracy and speed with respect to existing models such as SchNet and DCF in predicting the forces of various 
(a)

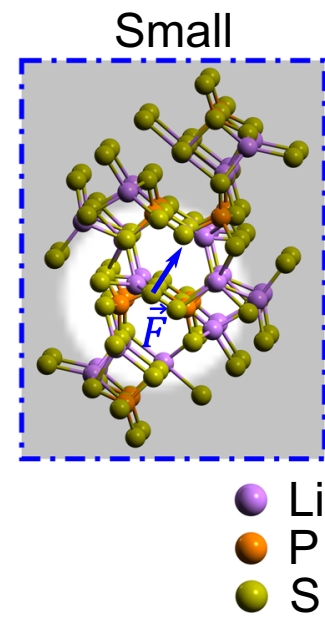

(b)

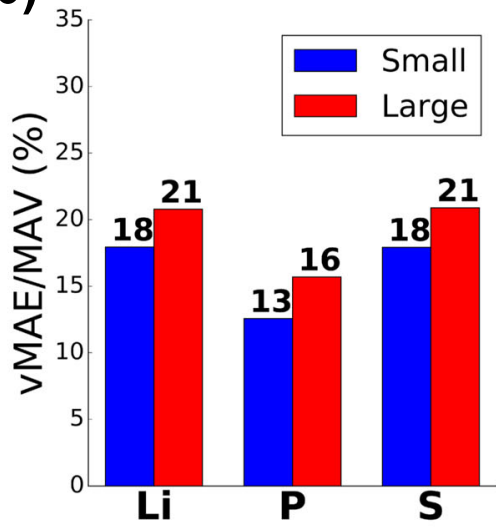

(d)

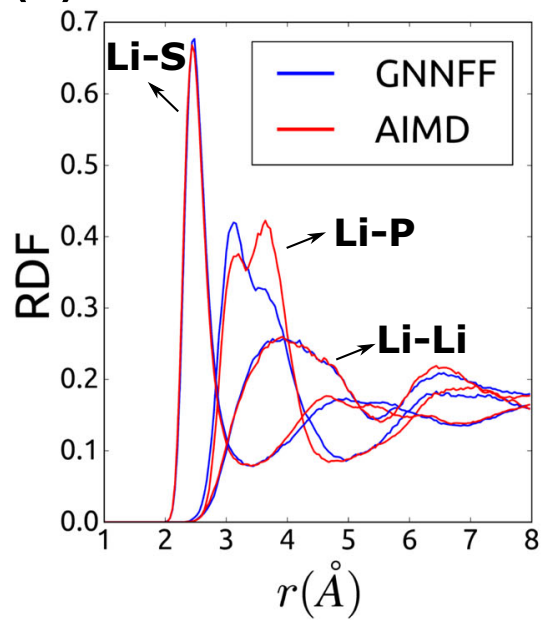

Large

GNNFF

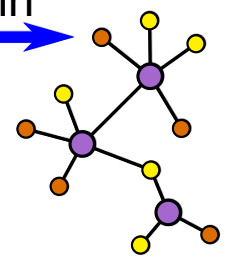

Predicti

(c)

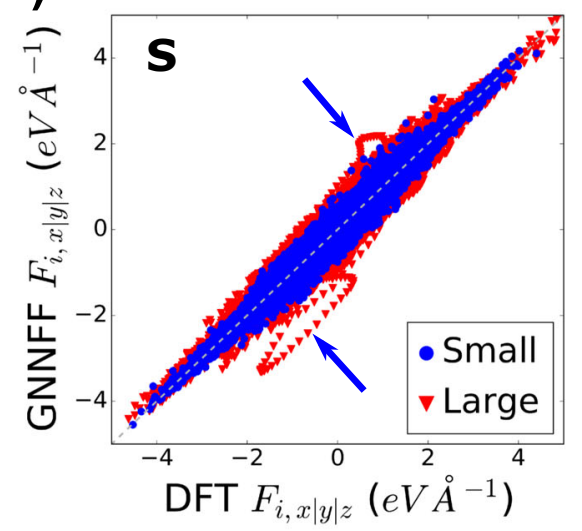

(e)

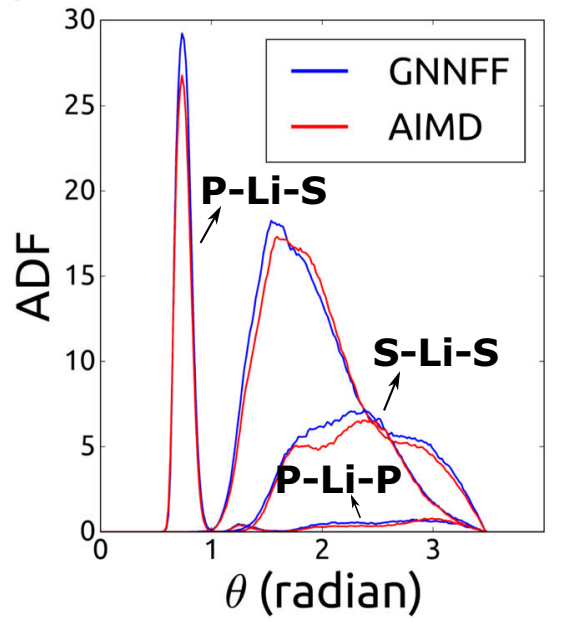

Fig. 4 Evaluating GNNFF scalability on AIMD trajectories of $\mathrm{Li}_{7-x} \mathbf{P}_{3} \mathbf{S}_{11}$ with differing $\mathbf{x}$ values. a GNNFF is trained on "Small" trajectory $(x=$ $0.25)$ forces and tested on "Large" trajectory $(x=0.125)$ forces b Comparison of GNNFF performance when evaluated on "Small" trajectory forces not used for training vs "Large" trajectory forces. c GNNFF-predicted vs DFT-calculated force correlation plots of S for both "Small' and "Large" systems. Blue arrows indicate significant deviations of ML-predicted values from DFT-calculated values. Comparison of the $\mathbf{d}$ radial distribution functions of different element pairs involving $\mathrm{Li}$ and $\mathrm{e}$ angular distribution functions of different element triplets involving Li from GNNFF and AIMD generated "Large" trajectories. The second element is the central atom of the triplet.

systems ranging from simple organic molecules and complex solid-state systems. We further showed that GNNFF has good scalability and can accurately predict the forces of large systems even after being trained on forces from a smaller system. Finally, we validated the physical/chemical accuracy of GNNFF by showing that the MD simulation of $\mathrm{Li}_{7-x} \mathrm{P}_{3} \mathrm{~S}_{11}$ driven by GNNFFcalculated forces can quantitatively capture the dynamics of Li-ion diffusion. We envision that GNNFF will significantly accelerate 

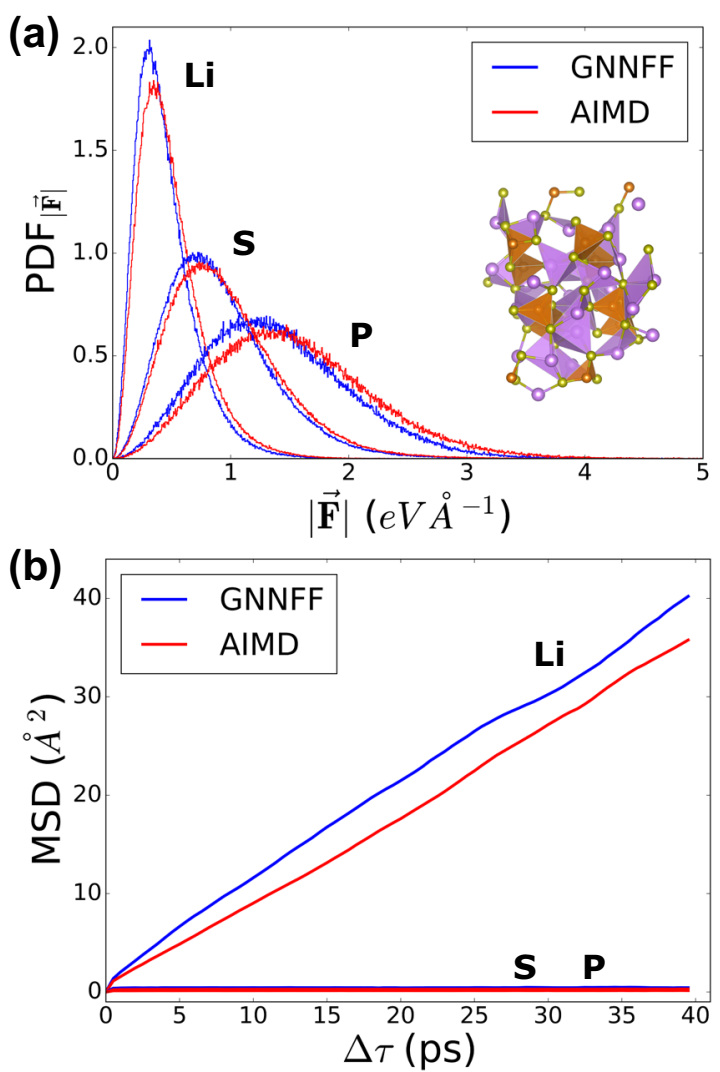

Fig. 5 Evaluating physical/chemical accuracy of GNNFF-generated trajectory of $\mathrm{Li}_{7-x} \mathrm{P}_{3} \mathrm{~S}_{11}$ in comparison to AIMD equivalent. a Probability distribution functions of force magnitudes for each element. Inset figure shows the structure of $\mathrm{Li}_{7-x} \mathrm{P}_{3} \mathrm{~S}_{11}$ obtained from the GNNFF-MD run after $50 \mathrm{ps}$. b Mean squared displacement of $\mathrm{Li}$, $S$, and $P$.

many different atomistic studies with its scalability, flexibility, and excellent force prediction accuracy.

\section{METHODS}

\section{Extracting reaction snapshots from $\mathrm{Al}_{2} \mathrm{O}_{3}-\mathrm{HF}$ MD trajectory}

The reaction snapshots in the $\mathrm{Al}_{2} \mathrm{O}_{3}-\mathrm{HF} \mathrm{MD}$ trajectory that correspond to the $\mathrm{HF}$ acid reacting at the surface of $\mathrm{Al}_{2} \mathrm{O}_{3}$ were extracted using an approach that combines molecular graph analysis and a $\mathrm{HMM}^{50,51}$. In this approach, snapshots of an MD trajectory are represented as graphs where each atom is connected to all neighboring atoms within a radial cutoff distance that depends on the covalent radii of the atoms. Here, we note that a connection between two atoms does not necessarily mean that there exists a bond between them. A connection is classified as a bond only after the connection consistently exists throughout a continuous time span. The level of consistency for which the connection must exist to be classified as a bond is determined by the HMM. This approach ensures that we do not mistake a cluster of atoms to be of the same molecule when they just happened to be close to each other due to thermal vibration or molecular collision. More details of this approach can be found in ref. ${ }^{39}$

\section{Generating $\mathbf{L i}_{\mathbf{7 - x}} \mathbf{P}_{\mathbf{3}} \mathrm{S}_{\mathbf{1 1}}$ ab initio data}

The AIMD simulations of $\mathrm{Li}_{7-x} \mathrm{P}_{3} \mathrm{~S}_{11}$ were performed using the Vienna $A b$ Initio Simulation Package $e^{2,3}$ with the project augmented wave method ${ }^{52}$ and Perdew-Burke-Ernzerhof generalized gradient approximation ${ }^{53}$. Two separate MD simulations were performed for a $1 \times 2 \times 1$ supercell ("Small") and a $1 \times 2 \times 2$ supercell ("Large"), both with periodic boundary conditions, at $520 \mathrm{~K}$ using a Nosé-Hoover thermostat. The initial structure for each run was obtained by relaxing and statically optimizing the defect-free structure prior to removing a $\mathrm{Li}^{+}$ion. $\Gamma$-point meshes of $3 \times 3 \times 3$ and $2 \times 2 \times 2$ were used respectively for the "Small" and "Large" simulations. The plane-wave cutoffs for both systems were set to $450 \mathrm{eV}$. In both runs, the temperature of the system was initialized at $100 \mathrm{~K}$ and then heated up to the target temperature of $520 \mathrm{~K}$ at a constant rate by rescaling the velocity over a time span of 4 ps. Each system was then equilibrated at the target temperature for another $4 \mathrm{ps}$. After equilibrating, the "Small" trajectory was simulated for $\sim 50 \mathrm{ps}$ while the "Large" trajectory was simulated for $\sim 14 \mathrm{ps,} \mathrm{both} \mathrm{using} \mathrm{a}$ timestep of $2 \mathrm{fs}$. Data used for training and testing the GNNFF only includes the trajectory generated after equilibration. In both trajectories, no breaking of the P-S bond was observed throughout the simulation.

\section{DATA AVAILABILITY}

The data that support the findings of this study are available from the corresponding author upon reasonable request. The training data for $\mathrm{Li}_{4} \mathrm{P}_{2} \mathrm{O}_{7}$ and $\mathrm{Al}_{2} \mathrm{O}_{3}$ - $\mathrm{HF}$ solidstate system is available through a Code Ocean compute capsule (https://doi.org/ 10.24433/CO.2788051.v1). The training data for the "Small" $\mathrm{Li}_{7-x} \mathrm{P}_{3} \mathrm{~S}_{11}$ system is available through Materials Cloud (https://doi.org/10.24435/materialscloud:66-ec).

\section{CODE AVAILABILITY}

The code for GNNFF is unavailable to the public due to proprietary reasons.

Received: 4 August 2020; Accepted: 19 April 2021; Published online: 21 May 2021

\section{REFERENCES}

1. Kresse, G. \& Hafner, J. Ab initiomolecular dynamics for liquid metals. Phys. Rev. $B$ 47, 558-561 (1993).

2. Kresse, G. \& Furthmüller, J. Efficient iterative schemes forab initiototal-energy calculations using a plane-wave basis set. Phys. Rev. B 54, 11169-11186 (1996).

3. Kresse, G. \& Furthmüller, J. Efficiency of ab-initio total energy calculations for metals and semiconductors using a plane-wave basis set. Comput. Mater. Sci. 6, 15-50 (1996).

4. Daw, M. S. \& Baskes, M. I. Embedded-atom method: Derivation and application to impurities, surfaces, and other defects in metals. Phys. Rev. B 29, 6443-6453 (1984).

5. Abell, G. Empirical chemical pseudopotential theory of molecular and metallic bonding. Phys. Rev. B 31, 6184-6196 (1985).

6. Stillinger, F. H. \& Weber, T. A. Computer simulation of local order in condensed phases of silicon. Phys. Rev. B 31, 5262-5271 (1985).

7. Tersoff, J. New empirical approach for the structure and energy of covalent systems. Phys. Rev. B 37, 6991-7000 (1988).

8. Tersoff, J. Empirical interatomic potential for carbon, with applications to amorphous carbon. Phys. Rev. Lett. 61, 2879-2882 (1988).

9. Brenner, D. W. Empirical potential for hydrocarbons for use in simulating the chemical vapor deposition of diamond films. Phys. Rev. B 42, 9458-9471 (1990).

10. Vashishta, P., Kalia, R. K., Rino, J. P. \& Ebbsjö, I. Interaction potential forSiO ${ }_{2}$ : a molecular-dynamics study of structural correlations. Phys. Rev. B 41, 12197-12209 (1990).

11. Rafii-Tabar, H. \& Sulton, A. P. Long-range Finnis-Sinclair potentials for f.c.c. metallic alloys. Philos. Mag. Lett. 63, 217-224 (1991).

12. Baskes, M. I. Modified embedded-atom potentials for cubic materials and impurities. Phys. Rev. B 46, 2727-2742 (1992).

13. van Duin, A. C. T., Dasgupta, S., Lorant, F. \& Goddard, W. A. ReaxFF: a reactive force field for hydrocarbons. J. Phys. Chem. A 105, 9396-9409 (2001).

14. Brenner, D. W. et al. A second-generation reactive empirical bond order (REBO) potential energy expression for hydrocarbons. J. Phys.: Condens. Matter 14, 783-802 (2002)

15. Jelinek, B. et al. Modified embedded atom method potential for $\mathrm{Al}, \mathrm{Si}, \mathrm{Mg}, \mathrm{Cu}$, and Fe alloys. Phys. Rev. B 85, 245102 (2012).

16. Harrison, J. A. et al. Review of force fields and intermolecular potentials used in atomistic computational materials research. Appl. Phys. Rev. 5, 031104 (2018).

17. Buckingham, R. A. The classical equation of state of gaseous helium, neon and argon. Proc. R. Soc. Lond. A 168, 264-283 (1938).

18. Tuckerman, M., Laasonen, K., Sprik, M. \& Parrinello, M. Ab initio molecular dynamics simulation of the solvation and transport of hydronium and hydroxyl ions in water. J. Chem. Phys. 103, 150-161 (1995).

19. Chu, I.-H. et al. Insights into the performance limits of the Li7P3S11 superionic conductor: a combined first-principles and experimental study. ACS Appl. Mater. Interfaces 8, 7843-7853 (2016). 
20. Chang, D., Oh, K., Kim, S. J. \& Kang, K. Super-ionic conduction in solid-state Li7P3S11-type sulfide electrolytes. Chem. Mater. 30, 8764-8770 (2018).

21. Valentini, P., Schwartzentruber, T. E. \& Cozmuta, I. Molecular dynamics simulation of $\mathrm{O} 2$ sticking on $\mathrm{Pt}(111)$ using the ab initio based ReaxFF reactive force field. J. Chem. Phys. 133, 084703 (2010).

22. Boes, J. R., Groenenboom, M. C., Keith, J. A. \& Kitchin, J. R. Neural network and ReaxFF comparison for Au properties. Int. J. Quantum Chem. 116, 979-987 (2016).

23. Behler, J. \& Parrinello, M. Generalized neural-network representation of highdimensional potential-energy surfaces. Phys. Rev. Lett. 98, 146401 (2007).

24. Bartók, A. P., Payne, M. C., Kondor, R. \& Csányi, G. Gaussian approximation potentials: the accuracy of quantum mechanics, without the electrons. Phys. Rev. Lett. 104, 136403 (2010).

25. Meredig, B. et al. Combinatorial screening for new materials in unconstrained composition space with machine learning. Phys. Rev. B 89, 094104 (2014).

26. Ward, L. et al. A general-purpose machine learning framework for predicting properties of inorganic materials. npj Comput. Mater. 2, 16028 (2016).

27. Chmiela, $\mathrm{S}$. et al. Machine learning of accurate energy-conserving molecular force fields. Sci. Adv. 3, e1603015 (2017).

28. Schütt, K. et al. Schnet: a continuous-filter convolutional neural network for modeling quantum interactions. Adv. Neural Inform. Process Syst. 30, 992-1002 (2017).

29. Schütt, K. et al. Quantum-chemical insights from deep tensor neural networks. Nat. Commun. 8, 13890 (2017).

30. Schütt, K. T., Sauceda, H. E., Kindermans, P.-J., Tkatchenko, A. \& Müller, K.-R SchNet-a deep learning architecture for molecules and materials. J. Chem. Phys. 148, 241722 (2018).

31. Xie, T. \& Grossman, J. C. Crystal graph convolutional neural networks for an accurate and interpretable prediction of material properties. Phys. Rev. Lett. 120, 145301 (2018)

32. Handley, C. M. \& Popelier, P. L. A. Potential energy surfaces fitted by artificial neural networks. J. Phys. Chem. A 114, 3371-3383 (2010).

33. Behler, J. Atom-centered symmetry functions for constructing high-dimensional neural network potentials. J. Chem. Phys. 134, 074106 (2011).

34. Gastegger, M. \& Marquetand, P. High-dimensional neural network potentials for organic reactions and an improved training algorithm. J. Chem. Theory Comput. 11, 2187-2198 (2015)

35. Huan, T. D. et al. A universal strategy for the creation of machine learning-based atomistic force fields. npj Comput Mater. 3, 37 (2017).

36. Smith, J. S., Isayev, O. \& Roitberg, A. E. ANI-1: an extensible neural network potential with DFT accuracy at force field computational cost. Chem. Sci. 8, 3192-3203 (2017).

37. Kondor, R., N-body networks: a covariant hierarchical neural network architecture for learning atomic potentials. arXiv:1803.01588 (2018)

38. Zhang, L., Han, J., Wang, H., Car, R. \& E, W. Deep potential molecular dynamics: a scalable model with the accuracy of quantum mechanics. Phys. Rev. Lett. 120 143001 (2018).

39. Mailoa, J. P. et al. A fast neural network approach for direct covariant forces prediction in complex multi-element extended systems. Nat. Mach. Intell. 1, 471-479 (2019)

40. Krizhevsky, A., Sutskever, I. \& Hinton, G. E. Imagenet classification with deep convolutional neural networks. Adv. Neural Inform, Process Syst. 25, 1097-1105 (2012).

41. Niepert, M., Ahmed, M. \& Kutzkov, K. Learning convolutional neural networks for graphs. International conference on machine learning. PMLR 48, 2014-2023 (2016).

42. Zhang, L. et al. End-to-end symmetry preserving inter-atomic potential energy model for finite and extended systems. Adv. Neural Inform. Process. 31, 4436-4446 (2018).

43. Chen, C., Ye, W., Zuo, Y., Zheng, C. \& Ong, S. P. Graph networks as a universal machine learning framework for molecules and crystals. Chem. Mater. 31, 3564-3572 (2019).

44. Xie, T. et al. Graph dynamical networks for unsupervised learning of atomic scale dynamics in materials. Nat. Commun. 10, 2667 (2019).

45. Park, C. W. \& Wolverton, C. Developing an improved crystal graph convolutional neural network framework for accelerated materials discovery. Phys. Rev. Mater. 4, 063801 (2020).

46. Ramakrishnan, R. et al. Quantum chemistry structures and properties of 134 kilo molecules. Sci. Data 1, 140022 (2014).

47. Hochreiter, S. \& Schmidhuber, J. Long short-term memory. Neural Comput 9 1735-1780 (1997).

48. Vandermause, J. et al. On-the-fly active learning of interpretable Bayesian force fields for atomistic rare events. npj Comput. Mater. 6, 20 (2020).
49. Li, W., Ando, Y., Minamitani, E. \& Watanabe, S. Study of Li atom diffusion in amorphous Li3PO4 with neural network potential. J. Chem. Phys. 147, 214106 (2017)

50. Wang, L. P. et al. Discovering chemistry with an ab initio nanoreactor. Nat. Chem. 6, 1044-1048 (2014).

51. Wang, L.-P., McGibbon, R. T., Pande, V. S. \& Martinez, T. J. Automated discovery and refinement of reactive molecular dynamics pathways. J. Chem. Theory Comput. 12, 638-649 (2016).

52. Kresse, G. \& Joubert, D. From ultrasoft pseudopotentials to the projector augmented-wave method. Phys. Rev. B 59, 1758-1775 (1999).

53. Perdew, J. P., Burke, K. \& Ernzerhof, M. Generalized gradient approximation made simple. Phys. Rev. Lett. 77, 3865-3868 (1996).

\section{ACKNOWLEDGEMENTS}

This work was performed in and funded by Bosch Research and Technology Center. This work was partially supported by ARPA-E Award No. DE-AR0000775. This research used resources of the Oak Ridge Leadership Computing Facility at Oak Ridge National Laboratory, which is supported by the Office of Science of the Department of Energy under Contract DE-AC05-00OR22725. C.W.P. and C.W. also acknowledge financial assistance from Award No.70NANB14H012 from US Department of Commerce, National Institute of Standards and Technology as part of the Center for Hierarchical Materials Design (CHiMaD) and the Toyota Research Institute (TRI). The authors also thank Eric Isaacs and Yizhou Zhu for helpful discussion.

\section{AUTHOR CONTRIBUTIONS}

J.P.M. conceived the project and jointly developed the method with C.W.P. C.W.P. implemented the GNNFF model with help from JPM and M.K. M.K., C.W.P., and J.P.M. generated the data that were used for evaluations. J.V., J.P.M., and C.W.P. built the GNNFF MD engine. C.W.P. performed the GNNFF MD study and error analysis with help from J.P.M., M.K. and B.K. B.K. mentors the research at Bosch and is the primary academic advisor of J.V. C.W. is the primary academic advisor for C.W.P. C.W.P. wrote the manuscript. All authors contributed to manuscript preparation.

\section{COMPETING INTERESTS}

The authors declare no competing interests.

\section{ADDITIONAL INFORMATION}

Supplementary information The online version contains supplementary material available at https://doi.org/10.1038/s41524-021-00543-3.

Correspondence and requests for materials should be addressed to B.K. or J.P.M.

Reprints and permission information is available at http://www.nature.com/ reprints

Publisher's note Springer Nature remains neutral with regard to jurisdictional claims in published maps and institutional affiliations.

\footnotetext{
Open Access This article is licensed under a Creative Commons Attribution 4.0 International License, which permits use, sharing, adaptation, distribution and reproduction in any medium or format, as long as you give appropriate credit to the original author(s) and the source, provide a link to the Creative Commons license, and indicate if changes were made. The images or other third party material in this article are included in the article's Creative Commons license, unless indicated otherwise in a credit line to the material. If material is not included in the article's Creative Commons license and your intended use is not permitted by statutory regulation or exceeds the permitted use, you will need to obtain permission directly from the copyright holder. To view a copy of this license, visit http://creativecommons. org/licenses/by/4.0/.
}

(c) The Author(s) 2021 